\title{
PIONEER
}

VOLUME 13, Issue 1, June 2021: 122-133

\section{REVEALING THE PATTERNS OF SEQUENCE ORGANIZATION ON BLACKPINK INTERVIEW AT ZACH SANG SHOW}

\author{
${ }^{1}$ Inkarizki Swedianisa Amalia* \\ ${ }^{1}$ Universitas Negeri Surabaya \\ 1amaliainka27@gmail.com
${ }^{2}$ Lina Purwaning Hartanti
${ }^{2}$ Universitas Negeri Surabaya
2linapurwaning@unesa.ac.id

*Corresponding author: amaliainka@gmail.com

$\begin{array}{llll}\text { Received } & \text { : March 10, 2021 } & \text { Revised } & \text { : May 16, 2021 } \\ \text { Accepted } & \text { : May 16, 2021 } & \text { Published } & \text { : June 30, 2021 }\end{array}$

\begin{abstract}
Intentionally or not, a conversation between two people or more is a common thing around us. This research will discuss the patterns of sequence organization in the conversation using the qualitative method. The data in this research were words, phrases, sentences in the form of spoken language taken from Youtube entitled "Blackpink Talks 'Kill This Love', Coachella \& How They Formed" which premiered on April 17, 2019. The research findings describe the patterns of sequence organization in conversation which consist of adjacency pairs, preference organization, sequence expansion, repair, and topic management. From 14 data, we found 17 adjacency pairs, which can be categorized into 6 types. The type of adjacency pair that is often asked by FPP is question-answer. There were 9 responses from SPP that showed preferred and 8 others indicated dispreferred and 4 repair sessions where all were initiated by other speakers. In addition, there are 8 expansion sequences which only 1 denotes pre-expansion, 6 denotes insert expansion, and one more denotes post expansion. From the patterns formed in the conversation, finally FPP can bring the flow of the conversation and produce 8 topics. FPP begins the conversation by asking about Blackpink performance at Coachella, then interspersed by discussing each member's life, how their initial meeting, what is their motivation, and talking about songs and choreography.
\end{abstract}

Keywords: Blackpink, interview, sequence organization in conversation, Zach Sang Show

\section{INTRODUCTION}

All human activities must require interaction and communication. In society, when a group of housewives buy vegetables in front of a neighbor's house, they have conversation with vegetable sellers to make offers. In another case, learning at school can be successful because of the interaction and conversation between the teacher and the 
students. Conversation is also a bridge to socialize with people. For example, some people, when accidentally meet, decide to greet each other to maintain their friendship. Conversation analysis is an approach that studies the interaction of speech through the sociological point of view sparked by Garfinkel (1967). Within the scope of conversation analysis, there is a term called sequence organization that refers to the form of social action (Liddicoat, 2007). Like the approach previously mentioned, participants show their actions through oral communication in a conversation. This is the idea of how some actions affect another action and determine the next action.

Over time, the development of media that involves social life and various cultures has garnered a lot of enthusiasts ( Khalid, Ahmed, Mufti, Asst, S. \& Asst,. 2015). not only in terms of domestic culture, but also in terms of foreign culture like the Korean Wave. in addition to treasuring the song and the album, many fans keep wondering about the personality of their favorite idol when interacting with other people. Since the K-pop world is more complicated and sensitive, it is used solely to ensure that people won't feel disappointed for supporting a particular artist. The high public interest in K-pop artists has made several media from various countries eager to collaborate and invite the artists to various radio stations and variety shows. This phenomenon brings an interesting point because the program must contain conversations which fulfill the fans' desire to show a more relaxed artist personality according to everyday life. ( Lundell, A. K., \& Ekstrom, 2010).

There are many channels or tv programs that invite K-pop artists as the guest stars. However, the researcher chose the interview video from Zach Sang Show YouTube channel since it hit $5.8 \mathrm{M}$ views and got good responses. This interview video also coincided when Blackpink participated as the first female K-Pop group in the Coachella festival so that it attracted a lot of public attention. Therefore, the subjects of the study are Zach as the interviewer and Blackpink members; Jisoo, Jennie, Rose and Lisa as the interviewees. The context of the data was dialogues between the interviewer and the interviewees.

The result of the study has a wider scope that tries to uncover the relative position of utterances or actions that affect the topic of conversation. In addition, it has been observed that not much about sequence organization has been done in the in-depth discussion, so it constitutes the gap which this study has come to fill. Therefore, the 
researcher formulated the research questions like the following: (1) What are the sequence organization patterns that occurred in the interview? (2) How does the interviewer switch topic conflicts during the interview? The results of this study could be a guideline for people who have interest in broadcasting especially in conducting interviews. Besides, they will master the design of effective topics of conversation to extract information from interviewees.

\section{REVIEW OF LITERATURE}

\section{Sequence Organization}

The basis of sequence organization in social interaction is adjacency pair, sequence expansion, preference organization, and repair session.

\section{Adjacency Pairs}

Adjacency pair organization is the automatic sequences in conversation produced by FPP [First Pair Part] and SPP [Second Pair Part] that come from the same pair type. In short, when the FPP asks "Do you want some tea or coffee?" then the SPP replied "I don't drink coffee". Regardless of the SPP response, as long as it is still related to the FPP question, it is still an adjacency pair. Thus, this term also shows a fundamental thing in the conversation. Levinson (1983) categorizes the types of adjacency pairs into greetings, questions-answers, goodbyes, offer-acceptance, assessment-agreement, compliment-acceptance, complain-apology, blame-denial, and warning-acknowledgment.

\section{Preference Organization}

As explained above, FPP and SPP are one unit in forming the adjacency pair type. However, in this section, there are preference terms that are devoted to SPP. Preferences are the SPP's freedom in responding to FPP, which is not determined through social status or power but rather preferred and dispreferred (Levinson, 1983). In short, when FPP makes a request to the SPP, there are two possibilities that occur; acceptance or rejection. He further makes the response correlation of adjacency pair seconds as follows: 
Table 1. Preference Organization

\begin{tabular}{llllll}
\hline First Parts & Request & $\begin{array}{l}\text { Offers/ } \\
\text { invitation }\end{array}$ & Assessment & Question & Blame \\
\hline $\begin{array}{l}\text { Second Parts } \\
\text { Preferred: }\end{array}$ & Acceptance & Acceptance & Agreement & $\begin{array}{l}\text { Expected } \\
\text { answer }\end{array}$ & Denial \\
& Refusal & Refusal & Disagreement & Unexpected & Admission \\
& & & & answer & (or non-) \\
\hline
\end{tabular}

In addition, the specified and turn shape characteristics are expressed in several ways such as delay, preface, account, and declination component. Delay is done by giving pauses to the conversation in order for the speaker to think of an appropriate answer before speaking. The preface can be expressed in words of appreciation if it is an invitation and an offer. While the account is a preferred type that expresses a reason directly and precisely, Declination components can also be used after delay and preface, so the declination component reinforces the intent of dispreferred that has been expressed by the second speaker.

\section{Sequence Expansion}

Such expansion involves additional participation by the speakers through additional turns that do not include the core turn. This expansion takes place in three possible places permitted by the two round units: before the first pair part (FPP) which is called pre-expansion; between FPP and the second pair part (SPP) of the base adjacency, called insertion expansion; and after FPP which is called post-expansion (Liddicoat, 2007).

Pre-expansion is used to describe actions such as pre-telling, invitations, requests, offers, etc., and it can be considered as preliminary actions (Liddicoat, 2007). He also adds that re-expansion affects the way in which subsequent talks will respond, and even the basis on which FPP will project depends on the results of the pre-expansion and some SPP responses. Insert expansion means the type of sequence expansion that can occur between an FPP and an SPP, and this model is quite often encountered. Insert expansions can be related to either the FPP that has expressed its adjacency pairs in which they are inserted which are called pre- 
second insert expansions, or they may address some issues arising from the FPP which precedes them, called post-first insert expansions.

Post-expansion takes a number of minimal post-expansion forms of sequence closing, two of the most common are: 'oh' and 'okay' which claim information receipt (Schegloff, 2007). While an assessment was taken up ordinarily by the FPP toward what the SPP has said or done in the prior turn. However, when a minimal post-expansion fails to close a sequence, the sequence will lead to non-minimal expansion. Non-minimal post-expansion also becomes the main alternative that the turn of FPP follows the SPP, so the turn generated is far from minimum expectations.

\section{Repair}

Repair leads to situations where there is a process that the speakers can do to change and improve their words. If a speaker encounters a problem in his words, he is able to do repairs on his own consciousness or called self-initiated self-repair. However, it may be initiated by another person or recipient called selfinitiated other-repair. In addition, if the recipient's speaking and repair are done by the speaker, it is called other-initiated self-repair, and if the recipient justifies his own words, it is called other-initiated other-repair (Liddicoat, 2007).

\section{Topic-proffering Sequences}

The type of adjacency pair in the form of questions, requests, offers, etc. is produced by one speaker by limiting the selection of topics, so this is also categorized in the action force of FPP (Mey, 2001). Speakers who control the topic usually bring the previous conversation to a conversation where there is a possibility of closing the topic sequence. A speaker also proposes a specific topic and invites the recipient to respond but does not actively launch or further develop the proposed topic. By offering a topic, the speaker makes two possibilities for the recipient to accept or reject (Schegloff, 2007).

According to Schegloff (2007), topic proffers can be categorized into two. First, the recipient-oriented topic that makes the recipient dominant in a discussion. The topic can involve something specific or based on the recipient's experience. In this case, the topic can develop due to an offer from FPP so that the SPP tends to carry the burden of discussion because they are the only informants and an object that can do so correctly. 
The second category is the topic that arises due to the type of yes / no question.that question arises the potential for further discussion of the topic.

\section{METHOD}

The phenomenon to be studied here is the interview between Zach as the interviewer and Blackpink members; Jisoo, Jennie, Rose and Lisa as the interviewees. This study reveals sequence organization and the management of topics which can be done by making the instrument in the form of an observation checklist. In the observation checklist, there are several tables that can simplify the classification of conversation patterns, such as adjacency pairs which have nine categories (Levinson, 1983), expansion sequences which have three categories (Liddicoat, 2007), and so on. In this case, this study applies a qualitative method since the purpose of this study consists of an inquiry that systematically uses a predetermined procedure to answer questions. The data in this research were words, phrases, sentences in the form of spoken language taken from https://youtu.be/W7f-6dVvw7I entitled “Blackpink Talks 'Kill This Love', Coachella \& How They Formed" which was premiered on April 17, 2019.

There is no physical contact between the researcher and the participants, as in the interview, but this is not a completely indirect method like novel analysis either (Cropley, 2019). The term virtual data is a method that allows the way data collection involves the use of the internet. Before doing some research, the researcher needs to collect the data. The stages of research procedure are choosing Blackpink as one of the K-pop groups that are being talked about by the public as well as the social media, then looking for Englishbased video interviews that invite Blackpink members as the interviewees, narrowing down some selected video interviews that both discuss Blackpink at the Coachella festival, deciding to choose an interview video from Zang Sang Show YouTube channel, downloading the chosen video from YouTube and also the script, watching the interview video which has been downloaded,cross-checking the video and the script in order to get accurate data,editing the script based on the spoken language of the video interview. The final stage is categorizing the data by using the observation checklist. 


\section{FINDINGS AND DISCUSSION}

\section{Findings}

The researcher has limited the data and found 17 adjacency pairs which can be summarized into 6 types; 1 greeting, 11 question - answer, 1 assessment - agreement, 2 compliment - acceptance, 1 complain - apology, and 1 goodbye. In addition, 3 types of sequence expansion are also found. The existence of pre-expansion in the form of pretelling affects changes in the topic of conversation. Meanwhile, the pattern of insert expansion in each adjacency pair indicate the repair session in conversation. These findings were based on the theory of Levison (1983) about the types of adjacency pairs and how SPP responds to FPP which indicates preference organization. On the other hand, Liddicoat (2007) and Schegloff (2007) support the findings of sequence expansion that formulate repair session and topic management.

\section{Adjacency Pair and Preference Organization}

Whenever speakers make an utterance, they expect certain answers. Obviously, when the second part is not fit with the first part, that cannot be called an adjacency pair. Talking about second part or SPP, it differentiates between the preferred social act and the dispreferred social act. Silence aims to avoid the direct refusal of the dispreferred social act and other indicators such as 'umm' and 'well'.

Datum 3 [0:06:33.935]

1 Zach : Okay Jenny, you learned English by going to school in NZ right, why go back to Korea and dive into the trainee program. Did you always want to do this?

2 Jennie : So, when I was in NZ and I live by myself like my family was living in Korea.

3 Zach : And you were so young.

4 Jennie : I know I turned 14 my mom wanted to move me to the states like Florida...

The situation in Datum 3 has two adjacency pairs and two preference organizations at the same time. Line 1 shows the type of question - answer proposed by FPP and the response from SPP is the unexpected answer since she does not directly answer questions that are considered dispreferred social act. The second adjacency pair is an assessment made by FPP, whereas SPP's responses indicate preferred ones because she uses less language to indicate agreement. 


\section{Sequence Expansion, Repair, and Topic in Conversation}

Discussing sequence expansion, it was previously mentioned that the patterns were interrelated with the existence of a repair session and how the topic of conversation was formed. Even so, FPP still plays an important role in changing topics so that topics can change at any time according to the type of adjacency pair proposed by FPP. Almost all the data show that the topic proffer occurred due to FPP's offer so that the SPP tends to carry the burden of discussion. However, in Datum 4 (lines 1-8), the formation of a topic occurs due to a yes / no question which allowed FPP to continue in a deeper discussion.

Datum 4-6 [0:08:34.096 - 0:09:38.077]

1 Zach : Do you remember the first time you met each other?

2 Rose : Definitely.

3 Lisa : Yeah.

4 Jisoo : (shaking her head)

5 Jennie : Yeah, talked about it, Rose joined us the last, a little girl from Australia she came along in us we're just connected, then

6 Rose : Singing together that day I brought a guitar and we ended up like jamming till like 5:00 a.m. ... (cutting)

7 Jennie : In the first day when we met.

8 Rose : I don't think we cared about what our neighbors thought it was an apartment sang till sunrise it was funny.

9 Zach : By the way it is crazy that you left New Zealand to go to Korea to join because did you audition yeah, I'll show you I'm...

10 Rose : Australia?

11 Zach : Oh, I'm so sorry.

12 Rose : No, it's okay cause I was born in New Zealand and I lived in Australia then I left.

13 Zach : Why'd you do the audition?

14 Rose : Because I think my dad sick of me like screaming every night at 12

a.m. just wanted to like get rid of me. My dad asked me, "do you want to take an audition?" and then they decided to be really support and I flew from Melbourne to Sydney to take the audition and I got it.

Line 1 shows pre-expansion in the form of pre-telling which is usually used to open a topic in a conversation. The responses from the four speakers were go-ahead answers because they immediately gave definite answers. Then the original adjacency pair which is the question - answer is responded alternately from lines 5-8 so that the appearance of the pre-expansion at the beginning does not change the origin of the adjacency pair that is formed. 
It is very interesting to discuss that in lines 10-11 there is an insert expansion as well as a repair session. The type of insert expansion that is formed is post-first insert expansion where in line 10 the speaker makes a complaint "Australia?" which eventually forms the expected answer from FPP. On the other hand, line 10 also indicates an otherinitiated-self-repair because there was a misinformation stated by FPP which was not realized until it was confirmed by SPP. Hence, in data 4-6 there is also a topic change that has been arranged by FPP as the interviewer. Lines 1-8 discuss how Blackpink members met each other, and lines 9-14 FPP focus on asking Rose about how she started her career.

To find out more clearly about the results of the sequence organization pattern that occurred in the interview between Zach Sang and Blackpink members as a whole can be seen in the brief table below.

Table 2. Sequence Organization Analysis

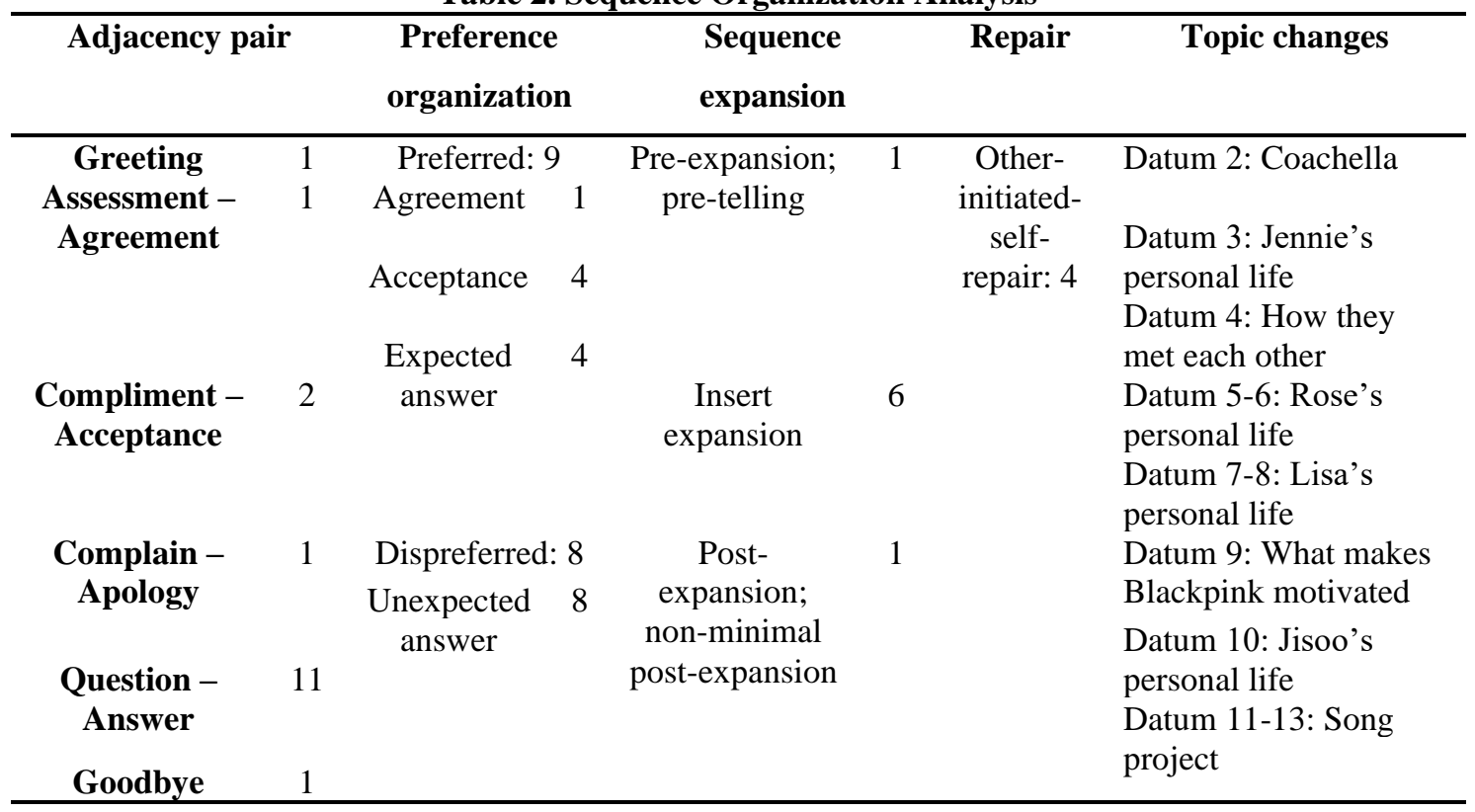

\section{Discussion}

The urgency of this study is to find out how the sequence of actions in the form of verbal speech is formed. Marta (2010) stated in CA that the action here occurs when one or both speakers start to realize it, so this is very different from speech act theory. Again, conversation analysis is not analyzing the grammar structure since it is based on conversations used daily (Hutchby, 2019). Instead of semantic approach, this study uses a more detailed approach and focuses more on naturally occurring data. conducting 
research using this approach takes time to investigate the footage and transcripts of the video. Besides, researchers should not add and change any word from the speaker because it will have an impact on the rules and hidden meanings in a sentence. In addition to the existing theories to support the results of research, concrete understanding is also needed to make it easier to read the situation.

After answering all the research problems, based on the findings, this study reveals that the occurrence of a sequence organization pattern in the interview is both predictable and unpredictable. However, if there is a planned pattern, it is indeed a script that is prepared by FPP only to regulate the flow of conversations such as creating a list of questions and some topics that will be discussed with SPP that indicate adjacency pairs. It should be noted that FPP does have control over the course of the topics and the duration of the interviews. However, all the patterns of the sequence organization are formed due to the response from SPP. As explained by Levinson (1983), the second part has the right to respond either preferred or dispreferred. Finally, FPP gets its information on the question or other adjacency pair model it poses.

As for preferred and dispreferred, Mudra (2018) in his study states that both preferred and dispreferred responses can not be interfered by the first speaker as such response relies upon the second speaker's sense. He also mentions in detail the kinds of preferred and dispreferred responses according to the form of adjacency pair and shows various indicators that illustrate the difference between the two and describes in detail each. In addition, insertion or generally known as insert expansion also often appears in adjacency pairs. In line with Liddicoat (2007) that among the three types of sequence expansion, insert expansion appears most often in conversations. However, in this study, researchers also found 2 other types that act as the opening and closing in an adjacency pair.

Although politeness universally indicates a good attitude, there are various connotations behind it as presented in the study by Jalilifar and Dinarvand (2019) regarding the reasons for speakers who remain polite in overcoming problems. He noted that cultural differences can also influence this. The use of this strategy may be somewhat ambiguous and complicated since it is an indirect statement. In conclusion, facial expressions play an important role in carrying out the concept of preference, whether in 
the form of acceptance or response directly or indirectly because not everyone is able to express and say the same thing.

The repair will occur when one of the speakers makes a mistake in conveying information. Hoey and Kendrick (2017) mentions in their study about three basic components that indicate repair session; trouble source, repair initiation, and repair solution. Another fact of the current study shows that an unpredictable situation is when one speaker makes a mistake and then he repairs himself or is corrected by other speakers. Repairing cannot be done intentionally to interrupt the conversation because it is influenced by the speakers' view in mind. In addition, there are 3 data which show that some speakers often experience the lost of vocabulary due to language barriers that are not the first language of the interviewees so that the repair session is often indicated by other speakers.

\section{CONCLUSIONS AND SUGGESTIONS Conclusions}

Basically, almost all the structure of the sequence organization pattern in conversation occurs naturally. It should be underlined that in this video interview, Zach, who plays the first part, holds the key to the continuity of the interview flow like a host in a broadcasting program. In essence, the interview has the aim to dig deeper into some information. Likewise, sequence organization enhances the conversation. The reason why the speakers perform sequence expansion and repair in adjacency pairs is based on reflex thoughts and experiences with an opinion. therefore, if these patterns occur in a conversation, they will not cause negative effects to each other.

\section{Suggestions}

The study about conversation analysis approach is always interesting to discuss since conversation is part of our daily activities. Therefore, the existence of sequence organization in conversation really supports how a conversation is generated in order to see how speakers understand and respond to each other according to their turn. The researcher expects the next researchers can investigate more deeply through this approach using a different context and find other reasons why speakers often use it. The researcher also hopes that the next researcher can examine the differences in culture in particular countries in conducting conversations or see from other points of view. Hopefully, the 
results of this study can be used as a reference and could be a guideline for people who have an interest in broadcasting especially in conducting interviews.

\section{REFERENCES}

Cropley, A. (2019). Introduction to Qualitative Research Methods. 0-188. https://doi.org/10.13140/RG.2.1.3095.6888

Garfinkel, H. (1967). Studies in Ethnomethodology. New Jersey: Prentice Hall.

Hoey, E. M., \& Kendrick, K. H. (2017). Conversation Analysis. Research Method in Psycholinguistics: A Practical Guide, 1-23.

Hoey, E. M., \& Kendrick, K. H. (2017). Conversation Analysis. Research Method in Psycholinguistics: A Practical Guide, 1-23.

Hutchby, I. (2019). Conversation Analysis. SAGE Research Methods Foundations, 1-21.

Jalilifar, A.R., \& Dinarvand, R. (2013). An analysis of Iranian EFL Learners' dispreferred responses in interactional discourse. The Journal of Teaching Language Skills (JTLS), 5(1), 19-44.

Khalid, M. Z., Ahmed, A., Mufti, S., Asst, S., \& Asst, S. (2015). Media and Development in Society: Continuity and Challenges. 20(11), 47-54. https://doi.org/10.9790/0837201124754

Lundell, A. K., \& Ekstrom, M. (2010). BEYOND THE BROADCAST INTERVIEW. Journalism Studies, (April). https://doi.org/10.1080/1461670X.2010.493328

L. Mey, J. (2001). Pragmatics: An Introduction (Second edition). London: Blackwell Publishing.

Levinson, S. C. (1983). Pragmatics (First). Cambridge: Cambridge University Press.

Liddicoat, A. J. (2007). An Introduction to Conversation Analysis. London/New York: Continuum.

Mudra, H. (2018). Adjacency Pairs as Uttered in the Conversations of Sofia Coppola's Lost in Translation Movir Script. Humanus, 126-137.

Schegloff, E. A., \& Angeles, L. (2017). Sequence Organization in Interaction: A Primer in Conversation Analysis. https://doi.org/10.1017/CBO9780511791208 